\title{
The Impact of Combining a Low-Tube Voltage Acquisition with Iterative Reconstruction on Total Iodine Dose in Coronary CT Angiography
}

\author{
Toon Van Cauteren, ${ }^{1}$ Gert Van Gompel, ${ }^{1}$ Kaoru Tanaka, ${ }^{1}$ Douwe E. Verdries, ${ }^{1}$ \\ Dries Belsack, ${ }^{1}$ Koenraad H. Nieboer, ${ }^{1}$ Inneke Willekens, ${ }^{1}$ Paul Evans, ${ }^{2}$ Sven Macholl, ${ }^{2}$ \\ Guy Verfaillie, ${ }^{1}$ Steven Droogmans, ${ }^{1}$ Johan de Mey, ${ }^{1}$ and Nico Buls ${ }^{1}$ \\ ${ }^{1}$ Radiology, Vrije Universiteit Brussel (VUB), Universitair Ziekenhuis Brussel (UZ Brussel), Laarbeeklaan 101, 1090 Brussels, Belgium \\ ${ }^{2}$ Imaging R\&D, GE Healthcare Life Sciences, The Grove Centre, Amersham, Buckinghamshire, UK \\ Correspondence should be addressed to Toon Van Cauteren; toon.van.cauteren@vub.ac.be
}

Received 31 January 2017; Revised 16 March 2017; Accepted 28 March 2017; Published 23 May 2017

Academic Editor: Atsushi Hirayama

\begin{abstract}
Copyright (C) 2017 Toon Van Cauteren et al. This is an open access article distributed under the Creative Commons Attribution License, which permits unrestricted use, distribution, and reproduction in any medium, provided the original work is properly cited.

Objectives. To assess the impact of combining low-tube voltage acquisition with iterative reconstruction (IR) techniques on the iodine dose in coronary CTA. Methods. Three minipigs underwent CCTA to compare a standard of care protocol with two alternative study protocols combining low-tube voltage and low iodine dose with IR. Image quality was evaluated objectively by the CT value, signal-to-noise ratio (SNR), and contrast-to-noise ratio (CNR) in the main coronary arteries and aorta and subjectively by expert reading. Statistics were performed by Mann-Whitney $U$ test and Chi-square analysis. Results. Despite reduced iodine dose, both study protocols maintained CT values, SNR, and CNR compared to the standard of care protocol. Expert readings confirmed these findings; all scans were perceived to be of at least diagnostically acceptable quality on all evaluated parameters allowing image interpretation. No statistical differences were observed (all $p$ values $>0.11$ ), except for streak artifacts $(p=0.02)$ which were considered to be more severe, although acceptable, with the $80 \mathrm{kVp}$ protocol. Conclusions. Reduced tube voltage in combination with IR allows a total iodine dose reduction between 37 and 50\%, by using contrast media with low iodine concentrations of 200 and $160 \mathrm{mg} \mathrm{I} / \mathrm{mL}$, while maintaining image quality.
\end{abstract}

\section{Introduction}

Iodine based contrast agents, such as those used in CT and angiography, can cause contrast-induced nephropathy $(\mathrm{CIN})$, which is associated with increased mortality in atrisk patients with renal insufficiency. Several studies demonstrated that CIN incidence is related to the administered iodine load, which motivates the aim for the reduction of the total iodine dose (TID) [1-6]. In CT contrast studies, image quality largely depends on the contrast between enhanced and nonenhanced regions; hence a sufficient amount of contrast agent must be administered to the patient to assure a certain image quality. A reduction of total iodine dose, for example, by reducing the iodine concentration of the contrast media, can only be achieved if the loss of image contrast is compensated. By decreasing the acquisition tube voltage, the attenuation difference between enhanced and nonenhanced tissues increases because the X-ray output energy approaches the iodine k-edge of $33 \mathrm{keV}$. This increase in image contrast opens up the opportunity to decrease the required iodine dose [7-9]. Scanning at lower photon energies, however, introduces more noise into the images, since X-rays of lower energies are more easily absorbed and consequently less photons reach the detector [10,11]. This noise increase could be tackled by increasing the tube current, resulting in an increased radiation dose, which is unfavourable considering patient safety. An alternative way to reduce the noise is the use of recently introduced iterative reconstruction (IR) techniques. Instead of using an idealised imaging model like in traditional filtered back-projection (FBP), these IR 
TABLE 1: Technical scan and injection parameters of the different scan protocols.

\begin{tabular}{lccc}
\hline Scan parameters & Standard of care protocol & Protocol A & Protocol B \\
\hline Tube voltage $(\mathrm{kVp})$ & 120 & 80 & 700 \\
Tube current $(\mathrm{mA})$ & 235 & 28.8 & 375 \\
CTDI $_{\text {vol }}(\mathrm{mGy})$ & 28.8 & Cardiac helical & Cardiac helical \\
Scan type & Cardiac helical & 30 & 30 \\
Scan delay $(\mathrm{s})$ & 30 & ASiR $60 \%$ & ASiR $60 \%$ \\
Image reconstruction model & FBP & 160 & 200 \\
Contrast media concentration $(\mathrm{mg} \mathrm{I} / \mathrm{mL})$ & 320 & 60 & 60 \\
Injection volume $(\mathrm{mL})$ & 60 & 3 & 3 \\
Injection rate $(\mathrm{mL} / \mathrm{s})$ & 3 & 0.48 & 0.60 \\
Iodine delivery rate $(\mathrm{g} \mathrm{I} / \mathrm{s})$ & 0.96 & 240 & 300 \\
Total iodine dose $(\mathrm{mg} \mathrm{I} / \mathrm{kg})$ & 480 & & \\
\hline
\end{tabular}

$\mathrm{mg} \mathrm{I} / \mathrm{mL}$ : milligrams of iodine per milliliter.

$\mathrm{g} \mathrm{I} / \mathrm{s}$ : grams of iodine per second.

$\mathrm{mg} \mathrm{I} / \mathrm{kg}$ : milligrams of iodine per kilogram of body weight.

techniques use an imaging model that describes the image acquisition, including noise statistics, which makes these algorithms more efficient and opens up the possibility of noise reduction [12-17].

Our hypothesis is that the total iodine dose can be substantially reduced when a lower tube voltage of 80 or $100 \mathrm{kVp}$ is used in combination with iterative reconstruction ASiR at a high blending level of $60 \%$ in coronary CT angiography.

The impact of the combination of a reduced tube voltage and iterative reconstruction on iodine dose has been the subject of several studies [7, 9, 11, 18], but, to our knowledge, this combination has not previously been reported with contrast media iodine concentrations below $300 \mathrm{mg} \mathrm{I} / \mathrm{mL}$ for a coronary CT angiography protocol, while still providing images of diagnostic quality.

The objective of this study was to assess the impact of combining a low-tube voltage acquisition with iterative reconstruction in a coronary CT angiography (CCTA) protocol on the necessary iodine dose in a porcine model scanned at constant radiation dose. The specific combinations of contrast media iodine concentration and tube voltage (320 $\mathrm{mg} \mathrm{I} / \mathrm{mL}$ at $120 \mathrm{kVp}$ for the standard of care versus 160 and $200 \mathrm{mg} \mathrm{I} / \mathrm{mL}$ at, respectively, 80 and $100 \mathrm{kVp}$ for the study protocols) used in this study were selected from previous research in abdominal CT [19-21]. Based on these experiences and on literature [22], we selected a $60 \%$ blending level of ASiR reconstruction for this CCTA study.

\section{Materials and Methods}

2.1. Porcine Model. Pigs are known to have a similar heart function and blood circulation to humans [23, 24]. Three healthy female, naive Göttingen minipigs (Ellegaard, Dalmose, Denmark) with a mean weight of $40.2 \mathrm{~kg}$ (range $38.2-41.5 \mathrm{~kg}$ ) and a mean effective chest diameter of $24.6 \mathrm{~cm}$ (range: $23.9-25.3 \mathrm{~cm}$ ) were included in the study. Study approval was granted by the institutional ethical committee for animal experiments. Two weeks before the start of the study, a port-a-cath (PAC) unit (Power PAC II, $1.9 \mathrm{~mm}$, Smiths Medical, St Paul, MN, USA) was placed subcutaneously at the level of the left shoulder with a connection to the superior vena cava to allow repeated contrast media injections in a consistent way. The pigs were scanned during a four-month period with an interscan delay of at least 72 hours to avoid iodine retention bias. Anaesthesia was induced by an intramuscular injection of an anaesthetic cocktail (500 mg Zoletil 100, 6.25 mg Rompun, 1.25 mL Ketamine, and $2.5 \mathrm{~mL}$ Dolorex) at a dose of $0.05 \mathrm{~mL}$ per $\mathrm{kg}$ body weight.

2.2. Scan and Injection Protocol. Coronary CT angiography (CCTA) was performed in free breathing with heart rate monitoring by a retrospective ECG-gated helical scan, at phase $70-75 \%$, on a 64-slice multidetector CT scanner (Discovery 750HD, GE Healthcare, Waukesha, WI, USA). No medication was used to control the heart rate considering the relatively low and stable rate of mean $62 \pm 4 \mathrm{bpm}$ over the total scan period. A standard of care scan protocol was compared with two alternative study protocols (Table 1). The standard of care protocol was scanned at a tube voltage of $120 \mathrm{kVp}$ with standard FBP image reconstruction. Study protocol A was scanned at $80 \mathrm{kVp}$, whereas protocol B was scanned at $100 \mathrm{kVp}$. The images of both study protocols were reconstructed with the iterative reconstruction (IR) technique ASiR at $60 \%$. The tube current was adapted for the different scan protocols to result in an equal radiation dose $\mathrm{CTDI}_{\mathrm{vol}}$ of $28.8 \mathrm{mGy}$. This was selected based on the exposure conditions of the $80 \mathrm{kVp}$ tube potential scan. The tube current of the other scan sequences was adapted to result in the same $\mathrm{CTDI}_{\mathrm{vol}}$ value. All scan protocols were acquired with a slice thickness and increment of $0.625 \mathrm{~mm}$ and a 30-second scan delay after injection. The scan protocol order was randomly assigned and repeated 3 times on two pigs, with confirmation in a third pig, resulting in a total of 21 scans.

Iodixanol (VISIPAQUE, GE Healthcare, Cork, Ireland) with an iodine concentration of $320 \mathrm{mg} \mathrm{I} / \mathrm{mL}$ was used as the 
standard of care contrast media. The reduced iodine concentrations of protocols A $(160 \mathrm{mg} \mathrm{I} / \mathrm{mL})$ and $\mathrm{B}(200 \mathrm{mg} \mathrm{I} / \mathrm{mL})$ were achieved by formulating the contrast media with additional saline (Baxter, Deerfield, IL, USA). The different contrast media were administered through the port-a-cath unit at controlled room temperature $\left(20^{\circ} \mathrm{C}\right)$ using a dual head injector from Nemoto Kyorindo (Tokyo, Japan). Contrast media administration was performed at a constant injection rate $(3 \mathrm{~mL} / \mathrm{s})$, injection volume $(60 \mathrm{~mL}, \sim 1.5 \mathrm{~mL} / \mathrm{kg})$, and saline chaser $(30 \mathrm{~mL})$, resulting in three different iodine delivery rates (IDR) and total iodine doses (TID) (Table 1).

\subsection{Objective and Subjective Image Quality Parameters.} Image quality was objectively evaluated by measuring the average CT value in Hounsfield units (HU) and image noise by the standard deviation (SD) in circular regions of interest (ROIs). These measurements were performed in the proximal coronary segment of the main coronary arteries, the right coronary artery (RCA), the left circumflex artery (LCx), and the left anterior descending artery (LAD), and in the aorta and the heart muscle tissue next to the aortic valve. Coronary ROIs had a diameter of $1.5 \mathrm{~mm}$; the ROIs of the aorta and heart muscle tissue were $10 \mathrm{~mm}$ in diameter. The signal-tonoise ratio (SNR) and contrast-to-noise ratio (CNR) were calculated. SNR was calculated as the ratio between the mean CT value and the SD, while CNR was calculated as (mean CT value organ of interest - mean $\mathrm{CT}$ value heart muscle)/SD heart muscle.

Two independent blinded expert readers (eight and ten years of experience in cardiac CT) randomly scored the images on four image quality parameters (Table 2) based on reported cardiac CT quality studies [13, 19, 24].

2.4. Statistical Analysis. Data were analyzed using commercially available software (SPSS, version 14; SPSS, Chicago, IL, USA). Statistical analysis by a nonparametric Mann-Whitney $U$ test was performed to compare the objective measurements (CT signal, SNR, and CNR) of the standard of care protocol with the results of both study protocols. A Chi-square test was used to assess the results of the subjective evaluation by the two expert readers. For both statistical techniques, $p$ values less than 0.05 indicate a significant difference. Intrapig and interpig variability was tested by $95 \%$ confidence intervals and Mann-Whitney $U$ test.

\section{Results}

3.1. Objective Image Quality Parameters. Objective analysis of the data shows no major differences between the mean measured CT values and calculated SNR and CNR of the standard of care protocol and both study protocols (Table 3 and Figures 1-3). The CT signal is slightly but not significantly lower in the coronary arteries after a CCTA with both study protocols as compared to the standard of care acquisition (Figure 1). For example, the mean coronary enhancement of the RCA is $268.3 \pm 14.2 \mathrm{HU}(p=0.14)$ for protocol A, 250.7 \pm $14.8 \mathrm{HU}(p=0.05)$ for protocol B, and $301.5 \pm 15.8 \mathrm{HU}$ for the standard of care protocol. Similar CT values are measured in

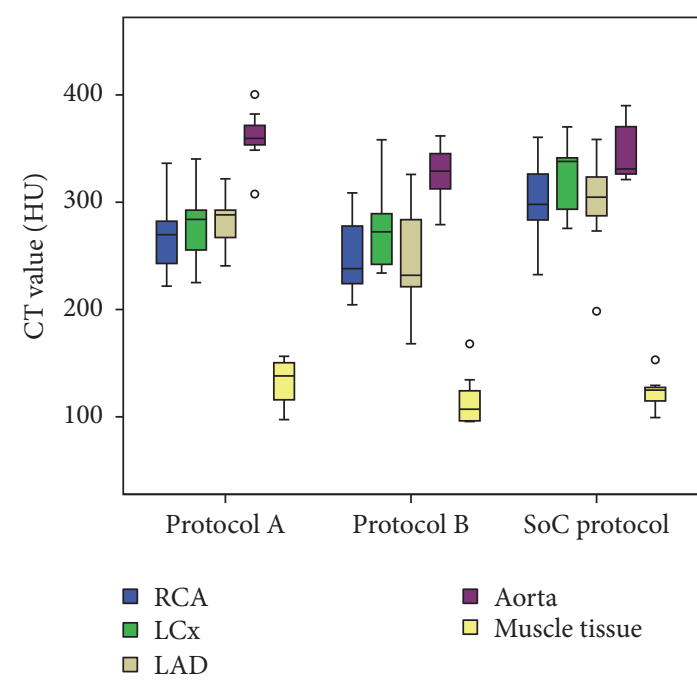

FIGURE 1: CT values for the right coronary artery (RCA), left circumflex artery (LCx), left anterior descending artery (LAD), aorta, and muscle tissue for both study protocols and the standard of care protocol. Boxplots display the median (middle bar), upper, and lower quartiles, while the whiskers (vertical line) indicate the variability outside the upper and lower quartiles. Mild outliers are displayed as o. No significant differences were found between the CT values of the study protocols and the standard of care protocol for any of the coronary arteries.

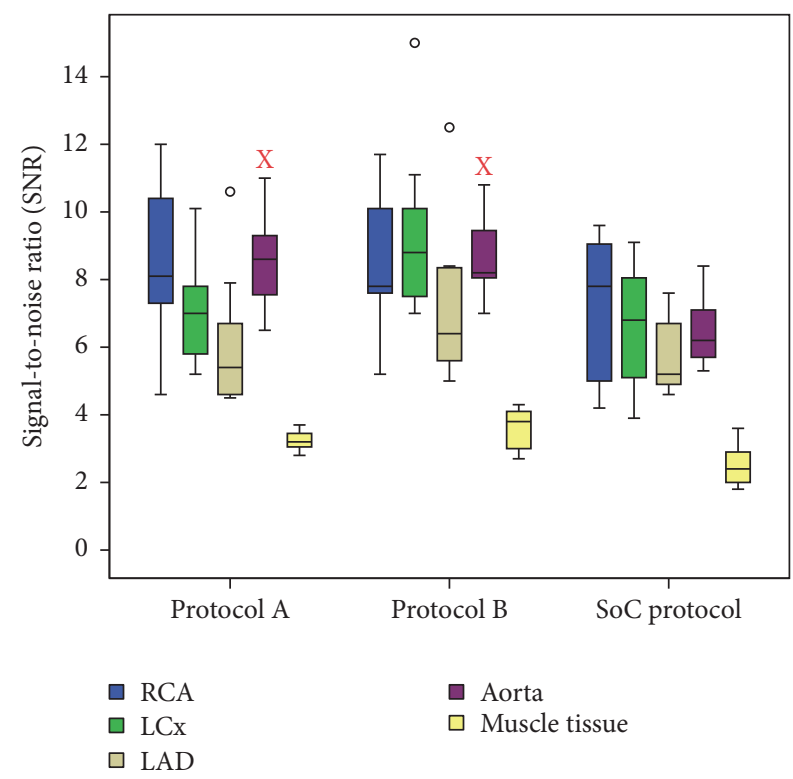

FIGURE 2: Signal-to-noise ratio for the right coronary artery (RCA), left circumflex artery (LCx), left anterior descending artery (LAD), aorta, and muscle tissue for both study protocols and the standard of care protocol. Boxplots display the median (middle bar), upper, and lower quartiles, while the whiskers (vertical line) indicate the variability outside the upper and lower quartiles. Mild outliers are displayed as $\mathrm{o}$. $\mathrm{X}$ indicates that there is a significant difference with the objective parameter of the standard of care protocol. 


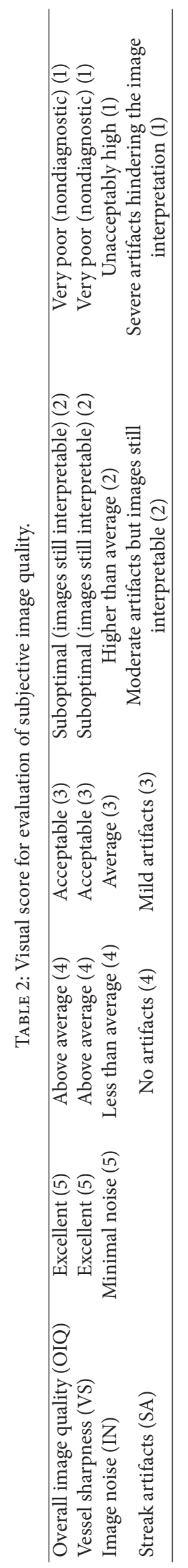




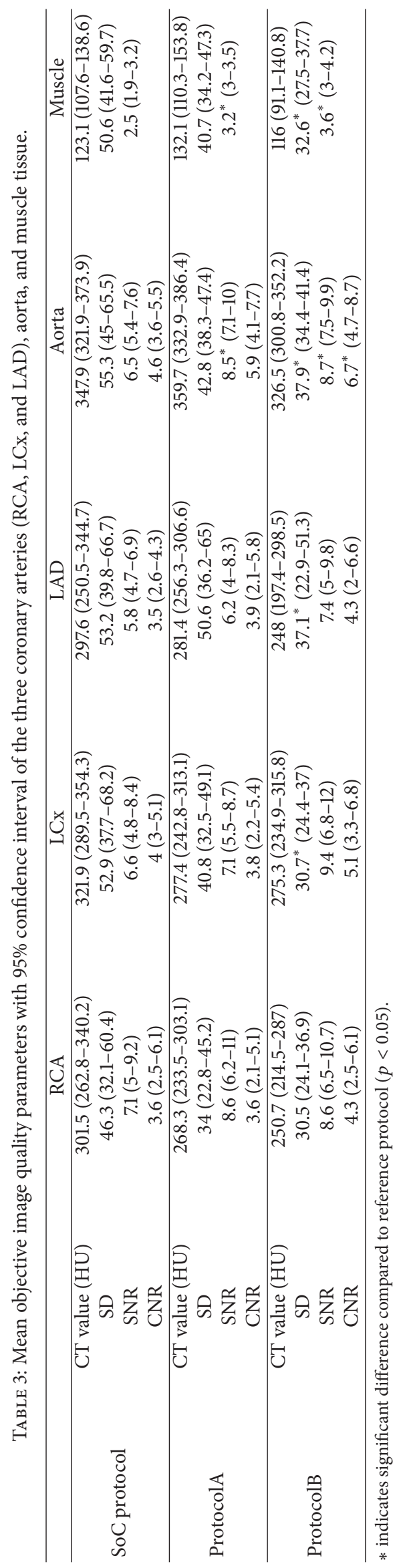


TABLE 4: Median subjective image quality parameters.

\begin{tabular}{lcccc}
\hline & Overall image quality (1-5) & Vessel sharpness (1-5) & Image noise (1-5) & Streak artifacts (1-4) \\
\hline SoC protocol & 4.0 & 4.0 & 4.0 & 4.0 \\
Protocol A & 4.0 & 3.5 & 3.5 & $3.0^{*}$ \\
Protocol B & 4.0 & 4.0 & 4.0 & 3.0 \\
\hline
\end{tabular}

$*$ indicates significant difference compared to reference protocol $(p<0.05)$.

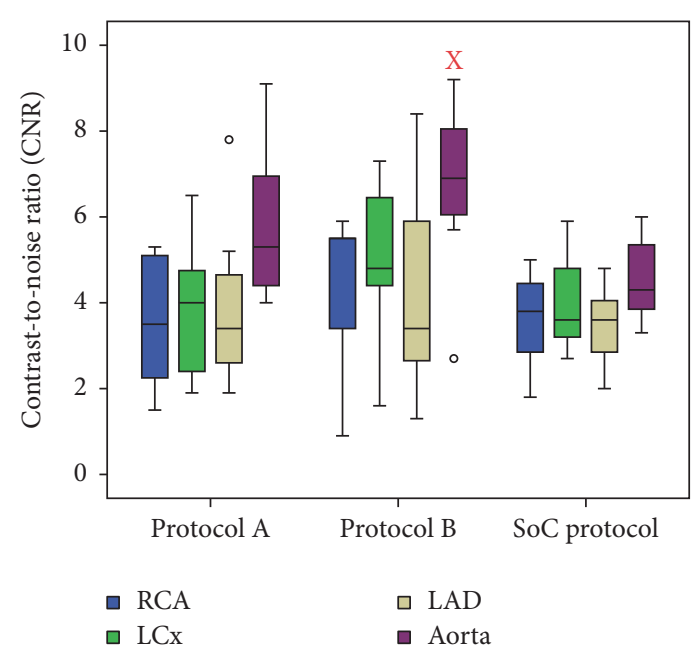

FIgURE 3: Contrast-to-noise ratio for the right coronary artery (RCA), left circumflex artery (LCx), left anterior descending artery (LAD), and aorta for both study protocols and the standard of care protocol. Boxplots display the median (middle bar), upper, and lower quartiles, while the whiskers (vertical line) indicate the variability outside the upper and lower quartiles. $\mathrm{X}$ indicates that there is a significant difference with the objective parameter of the standard of care protocol.

the other coronary arteries. The SNR and CNR of the study protocols are slightly but not significantly higher compared to the SNR and CNR of the standard of care protocol. For example, the mean SNR of the RCA is $8.6 \pm 1(p=0.48)$ for protocol A, 8.6 \pm 0.8 ( $p=0.41)$ for protocol B, and $7.1 \pm 0.9$ for the standard of care protocol (Figure 2). The mean CNR of the RCA is $3.6 \pm 0.6(p=0.8)$ for protocol A, $4.3 \pm 0.7(p=0.08)$ for protocol $\mathrm{B}$, and $3.6 \pm 0.5$ for the standard of care protocol (Figure 3). Similar results can be observed for the LCx and the LAD. Some significant differences are observed; for example, both study protocols result in significantly higher SNR for the aorta $(8.5 \pm 0.6(p=0.02)$ for protocol A and $8.7 \pm 0.5$ ( $p=0.02$ ) for protocol B) compared to the standard of care protocol $(6.5 \pm 0.5)$ (Figure 2). The mean aorta CNR of the images from protocol B $(6.7 \pm 0.8(p=0.04))$ is significantly higher compared to the mean aorta CNR of the standard of care protocol (4.6 \pm 0.4$)$ (Figure 3). The soft tissue image noise, measured as the SD of nonenhanced muscle tissue, is significantly lower in the images of protocol B $(32.6 \pm 2.1$ $(p=0.004))$, scanned with a tube voltage of $100 \mathrm{kVp}$ and with ASiR $60 \%$, compared to the image noise of the standard of care protocol (50.6 \pm 3.7$)$ (Table 3). For all three evaluated image quality parameters, no significant differences were observed between both pigs for any of the tested protocols (mean $p$ value was 0.24 ). Also, for the intrapig variability, all obtained results were within the $95 \%$ confidence intervals.

3.2. Subjective Image Quality Parameters. No significant differences for the overall image quality, vessel sharpness, and image noise are observed between the standard of care protocol and both study protocols. For example, the overall image quality for the standard of care protocol had a median score of 4 (range: $3-5)$, whereas it was 4 (range: $2-5$ ) for protocol A $(p=0.58)$ and 4 (range: $3-5)$ for protocol B $(p=0.29)$. Regarding streak artifacts, a significant increase was observed between the standard of care protocol (median score of 4 (range: $2-4)$ ) and protocol A (median score of 3 (range: $2-4)(p=0.021)$ ) (Figure 4$)$ but not with protocol B (median score of 3 (range: $2-4)(p=0.17)$ ). The images of all three scan protocols were evaluated by the observers to be of at least diagnostic image quality on all evaluated parameters (Table 4 and Figure 5).

\section{Discussion}

Several studies have demonstrated that contrast-induced nephropathy (CIN) incidence is related to the administered iodine dose. This motivates the aim for a reduction of the total iodine dose (TID) which can be beneficial for patient safety, especially for patients with a reduced kidney function [1-6].

The total iodine dose used for a coronary CTA, as reported in literature, is typically between 350 and $600 \mathrm{mgI} / \mathrm{kg}$, resulting in a coronary enhancement of 300-400 HU [25-27]. This corresponds well with the total iodine dose $(480 \mathrm{mg} \mathrm{I} / \mathrm{kg})$ of the standard of care protocol of this study, which uses a contrast media iodine concentration of $320 \mathrm{mg} \mathrm{I} / \mathrm{mL}$, resulting in a mean CT signal of $307 \mathrm{HU}$. Data presented in this study suggest that this concentration can be lowered down to 160 or $200 \mathrm{mg} \mathrm{I} / \mathrm{mL}$ (total iodine dose of 240 and $300 \mathrm{mg} / \mathrm{kg}$, resp.), when combined with reduced tube voltages of 80 and $100 \mathrm{kVp}$, respectively, and additional use of iterative reconstruction ASiR 60\%. Even with the reduced total iodine doses of the study protocols, the CT signal, SNR, and CNR were preserved compared to the standard of care CCTA protocol. A noteworthy result is the SNR of the LAD, which was slightly lower compared to the SNR of the RCA and LCx in all three scan protocols. Since the CT signal in the LAD was not inferior compared to the other coronary arteries, it is the slightly higher image noise that is responsible for the lower SNR. A possible explanation is that local noise levels are different in the LAD due to the surrounding tissue or due to the different location of the LAD in the scan field. 


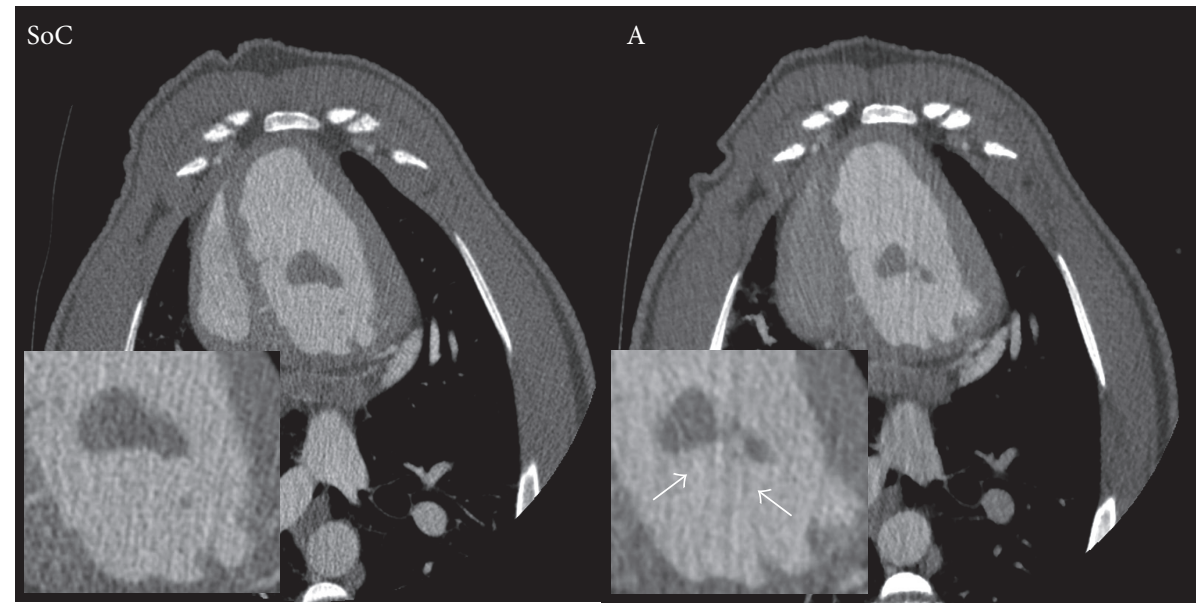

Figure 4: Images of the standard of care (SoC) protocol and study protocol A (A) are compared to demonstrate the significant higher chance on streak artifacts (white arrows) in images scanned at $80 \mathrm{kV}$ (protocol A) compared to $120 \mathrm{kV}$ images (SoC protocol).

The images of both study protocols received equal scores compared to the standard of care protocol's images considering overall image quality, vessel sharpness, and image noise by the expert readers. Streak artifacts were more severe in the images of protocol $\mathrm{A}$ in comparison with the images of the standard of care protocol; this appearance of streak artifacts is a well-known observation in low-tube voltage acquisitions $[28,29]$. No differences in the appearance of streak artifacts were found between the images of protocol B and the images of the standard of care protocol. This indicates that study protocol B, with a tube voltage of $100 \mathrm{kVp}$, may be preferred for patients with a larger chest diameter compared to study protocol $\mathrm{A}$, with a tube voltage of $80 \mathrm{kVp}$, which may be more suitable for thinner patients, because not only the appearance of streak artifacts but also the general image quality of low $\mathrm{kV}$ scans is affected by the object size [30]. All images of the three protocols were scored as at least diagnostically acceptable, even with the presence of increased streak artifacts in the images of protocol A which did not impair image interpretation.

From these results, we can conclude that the combination of a reduced tube voltage and the iterative reconstruction ASiR 60\% allows a substantial iodine dose reduction in CCTA without compromising diagnostic image quality in a porcine model. An iodine concentration reduction from $320 \mathrm{mg} \mathrm{I} / \mathrm{mL}$ to 200 or $160 \mathrm{mgI} / \mathrm{mL}$ corresponds with an iodine dose reduction in the order of $37 \%(100 \mathrm{kVp})$ to $50 \%(80 \mathrm{kVp})$. These results are well in agreement with the results described by Nakaura et al. [18] who achieved a similar iodine dose reduction in patients scanned at $80 \mathrm{kVp}$ in combination with a hybrid iterative iDose level of 60\% (Phillips Healthcare). The iodine dose reduction, in our study, was achieved by using contrast media with decreased iodine concentrations of 160 or $200 \mathrm{mg} \mathrm{I} / \mathrm{mL}$. These are remarkable lower iodine concentrations than typically reported in similar iodine dose reducing studies $[7,9,11,18]$ that used contrast media with iodine concentrations not lower than $300 \mathrm{mg} \mathrm{I} / \mathrm{mL}$. These subclinically low iodine concentrations are in strong contrast with the typically higher iodine concentrations used in clinical CCTA practice (between 320 and $400 \mathrm{mg} \mathrm{I/mL)} \mathrm{[25-}$ 27].

As the innovations in iterative reconstruction methods are still ongoing, we expect that more advanced reconstruction methods will result in further noise reduction and hence improved SNR and CNR levels. When SNR and CNR are relevant figures of merit for the diagnostic task, this gain in SNR or CNR could be converted to further reduction of iodine dose. On the other hand, when diagnostic quality is reflected by the $\mathrm{CT}$ value (HU) rather than SNR or CNR, further iodine dose reduction will affect diagnostic quality negatively, as iterative reconstruction methods have intrinsically no impact on the CT value. However, in that case, advanced reconstruction methods could compensate for the increased noise levels at lower tube potentials $(70 \mathrm{kVp})$ [31], which could be beneficial for small size patients and children with reduced kidney function [32]. Another beneficial effect of the use of iterative reconstruction methods is the possible reduction of radiation dose exposure, but this was not the aim of this study. Compared to our results, a scan protocol at lower radiation dose would have resulted in less drastic iodine dose reductions for images of equal quality.

For practical reasons, we used a retrospective ECGgated helical scan technique, which is typically related to a higher radiation dose than a prospective step and shoot technique [33]. This scan technique allowed retrospective manual adjustments of the ECG-based trigger points, which were sometimes suboptimal due to a disturbed ECG signal through the thick pig skin. Nevertheless, similar total iodine dose reduction results can be expected with other cardiac scan techniques.

Limitations of this study are mainly related to the porcine model. Despite the similar cardiac anatomy and function of the minipigs compared to humans, further validation of 


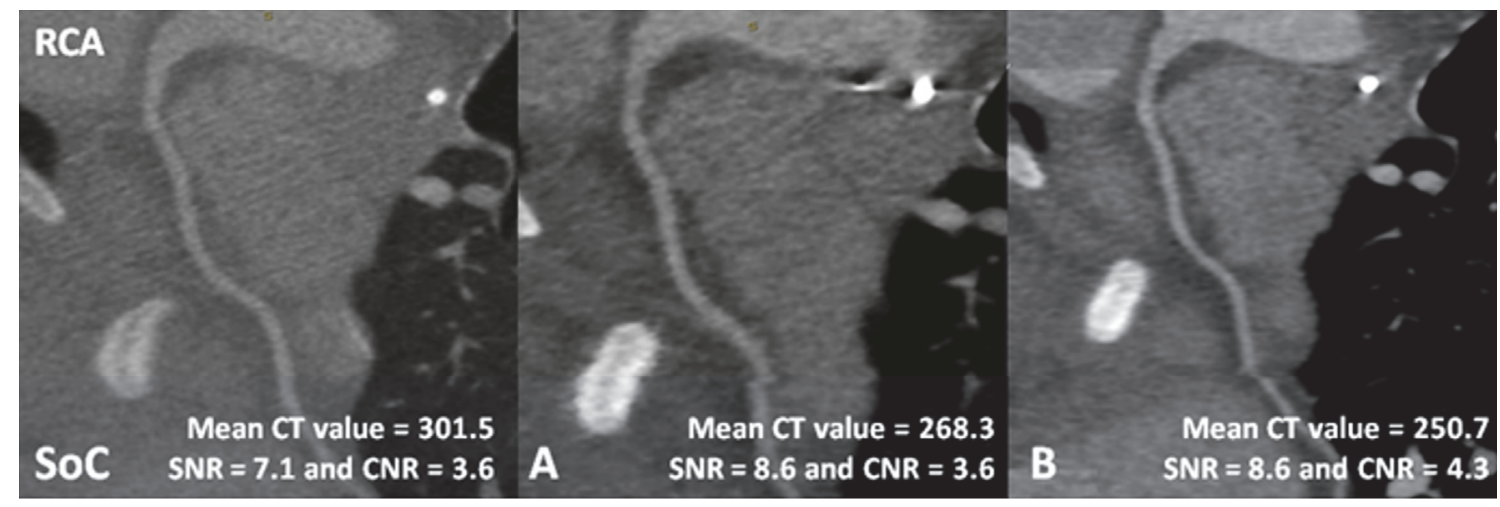

(a)

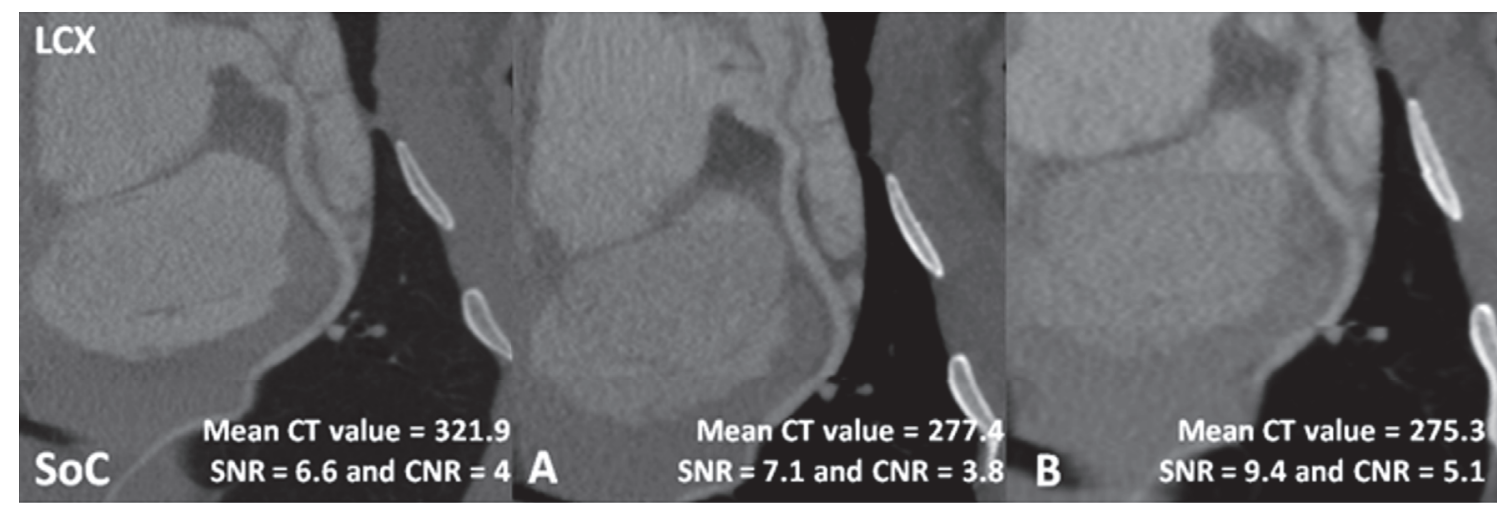

(b)

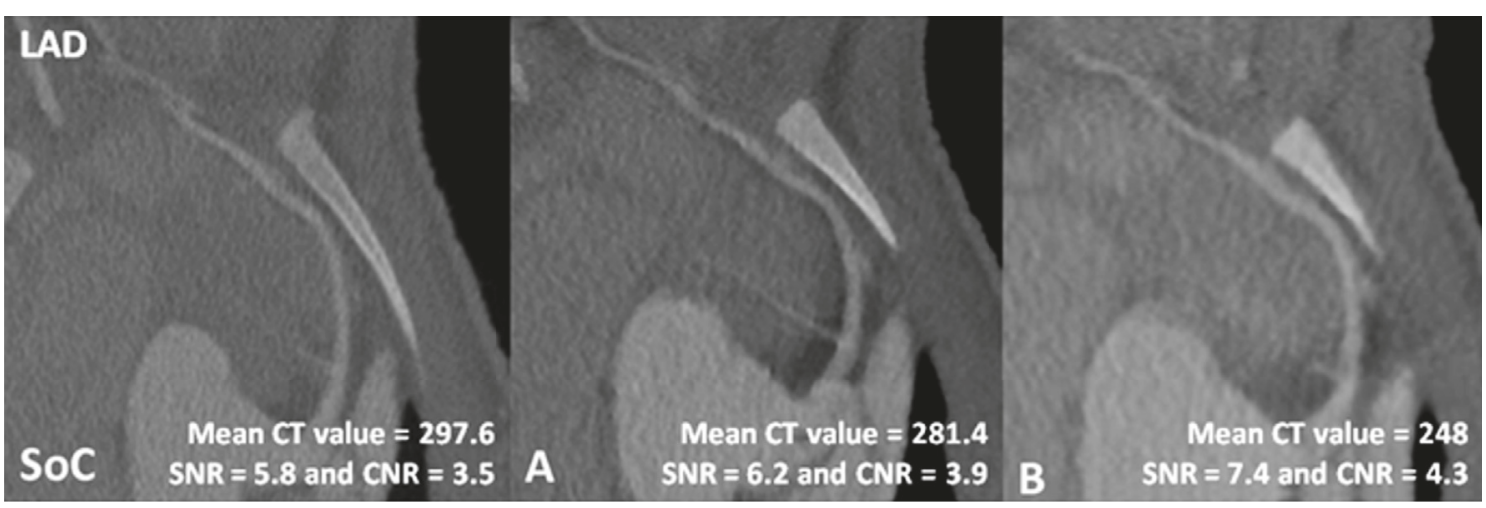

(c)

FIGURE 5: Images of the standard of care protocol and study protocols A and B with the mean CT value, SNR, and CNR of the right coronary artery (RCA) (a), left circumflex artery (LCx) (b), and the left anterior descending artery (LAD) (c).

the alternative study CCTA protocols in patient studies is required before being applied in clinical practice. Although a lot of experimental work to validate new CT technologies can be done in phantom studies, this is not always possible. For example, realistic phantom models that mimic the complex anatomy/physiology of the human heart and coronaries do exist but are rare and are usually confined to investigating a limited amount of physiological parameters [34, 35]. Therefore drastic changes of the scan or injection protocol, like the use of contrast media with subclinical iodine concentrations of 160 or $200 \mathrm{mgI} / \mathrm{mL}$, can be assessed in preclinical animal studies before applying them on patients. The minipigs used in this study were healthy animals with no presence of any coronary stenosis or atherosclerotic plaque. The diagnostic accountability of these pathologies or the ability to analyze the coronary plaque components was not tested in this study. Another limitation is the use of a porta-cath. The vast majority of CCTA patients have contrast media administered through a peripheral injection in the antecubital vein, while our study used a port-a-cath injection which delivers the contrast media directly to the superior vena cava. This difference in contrast media administration 
may have an impact on the required iodine dose to result in a sufficient coronary enhancement. However, a similar total iodine dose reduction, by combining a low-tube voltage acquisition with the iterative reconstruction $\mathrm{ASiR} 60 \%$, may be expected independent of the contrast media administration technique. For example, similar iodine dose reduction results were found in patients with an intravenous contrast media administration [18].

\section{Conclusion}

The results suggest that a significant total iodine dose reduction appears feasible in clinical CCTA by combining low-tube voltage acquisitions with iterative reconstruction techniques, from $480 \mathrm{mg} \mathrm{I} / \mathrm{kg}$ down to $300-240 \mathrm{mg} \mathrm{I} / \mathrm{kg}$ or about $37 \%(100 \mathrm{kVp})$ to $50 \%(80 \mathrm{kVp})$, by using contrast media with iodine concentrations of 200 and $160 \mathrm{mg} \mathrm{I} / \mathrm{mL}$, while maintaining image quality as found in this porcine study. Contrast media administration should be continuously reassessed in relation to the evolving CT technology.

\section{Conflicts of Interest}

The authors declare that there are no conflicts of interest regarding the publication of this paper.

\section{Acknowledgments}

This study has received funding by GE Healthcare Medical Diagnostics.

\section{References}

[1] L. Gruberg, G. S. Mintz, R. Mehran et al., "The prognostic implications of further renal function deterioration within 48 $\mathrm{h}$ of interventional coronary procedures in patients with preexistent chronic renal insufficiency," Journal of the American College of Cardiology, vol. 36, no. 5, pp. 1542-1548, 2000.

[2] Y. Zhao, Z. Tao, Z. Xu et al., "Toxic effects of a high dose of nonionic iodinated contrast media on renal glomerular and aortic endothelial cells in aged rats in vivo," Toxicology Letters, vol. 202, no. 3, pp. 253-260, 2011.

[3] E. Seeliger, M. Sendeski, C. S. Rihal, and P. B. Persson, "Contrast-induced kidney injury: mechanisms, risk factors, and prevention," European Heart Journal, vol. 33, no. 16, pp. 20072015, 2012.

[4] G. Marenzi, E. Assanelli, J. Campodonico et al., "Contrast volume during primary percutaneous coronary intervention and subsequent contrast-induced nephropathy and mortality," Annals of Internal Medicine, vol. 150, no. 3, pp. 170-177, 2009.

[5] R. Raju, A. G. Thompson, K. Lee et al., "Reduced iodine load with CT coronary angiography using dual-energy imaging: A prospective randomized trial compared with standard coronary CT angiography," Journal of Cardiovascular Computed Tomography, vol. 8, no. 4, pp. 282-288, 2014.

[6] P. Carrascosa, C. Capunay, G. A. Rodriguez-Granillo, A. Deviggiano, J. Vallejos, and J. A. Leipsic, "Substantial iodine volume load reduction in CT angiography with dual-energy imaging: insights from a pilot randomized study," International Journal of Cardiovascular Imaging, vol. 30, no. 8, pp. 1613-1620, 2014.
[7] T. Nakaura, K. Awai, N. Maruyama et al., "Abdominal dynamic CT in patients with renal dysfunction: contrast agent dose reduction with low tube voltage and high tube current-time product settings at 256-detector row CT,' Radiology, vol. 261, no. 2, pp. 467-476, 2011.

[8] K. T. Bae, "Intravenous contrast medium administration and scan timing at CT: considerations and approaches," Radiology, vol. 256, no. 1, pp. 32-61, 2010.

[9] A. B. Sigal-Cinqualbre, R. Hennequin, H. T. Abada, X. Chen, and J.-F. Paul, "Low-kilovoltage multi-detector row chest ct in adults: feasibility and effect on image quality and iodine dose," Radiology, vol. 231, no. 1, pp. 169-174, 2004.

[10] Z. Szucs-Farkas, S. Bensler, J. C. Torrente, J. L. Cullmann, P. Vock, and S. T. Schindera, "Nonlinear three-dimensional noise filter with low-dose CT angiography: effect on the detection of small high-contrast objects in a phantom model," Radiology, vol. 258, no. 1, pp. 261-269, 2011.

[11] A. E. Papadakis, K. Perisinakis, M. Raissaki, and J. Damilakis, "Effect of X-ray tube parameters and iodine concentration on image quality and radiation dose in cerebral pediatric and adult CT angiography: a phantom study," Investigative Radiology, vol. 48, no. 4, pp. 192-199, 2013.

[12] A. K. Hara, R. G. Paden, A. C. Silva, J. L. Kujak, H. J. Lawder, and W. Pavlicek, "Iterative reconstruction technique for reducing body radiation dose at CT: feasibility study," American Journal of Roentgenology, vol. 193, no. 3, pp. 764-771, 2009.

[13] G. S. Desai, R. N. Uppot, E. W. Yu, A. R. Kambadakone, and D. V. Sahani, "Impact of iterative reconstruction on image quality and radiation dose in multidetector CT of large body size adults," European Radiology, vol. 22, no. 8, pp. 1631-1640, 2012.

[14] M. Katsura, I. Matsuda, M. Akahane et al., "Model-based iterative reconstruction technique for radiation dose reduction in chest CT: comparison with the adaptive statistical iterative reconstruction technique," European Radiology, vol. 22, no. 8, pp. 1613-1623, 2012.

[15] D. Marin, R. C. Nelson, S. T. Schindera et al., "Low-tube-voltage, high-tube-current multidetector abdominal CT: improved image quality and decreased radiation dose with adaptive statistical iterative reconstruction algorithm-initial clinical experience," Radiology, vol. 254, no. 1, pp. 145-153, 2010.

[16] C. Gebhard, T. A. Fuchs, M. Fiechter et al., "Image quality of low-dose CCTA in obese patients: impact of high-definition computed tomography and adaptive statistical iterative reconstruction," International Journal of Cardiovascular Imaging, vol. 29, no. 7, pp. 1565-1574, 2013.

[17] M. J. Willemink, T. Leiner, P. A. de Jong et al., "Iterative reconstruction techniques for computed tomography part 2 : initial results in dose reduction and image quality," European Radiology, vol. 23, no. 6, pp. 1632-1642, 2013.

[18] T. Nakaura, M. Kidoh, N. Sakaino et al., "Low contrast- and low radiation dose protocol for cardiac CT of thin adults at 256row CT: usefulness of low tube voltage scans and the hybrid iterative reconstruction algorithm," International Journal of Cardiovascular Imaging, vol. 29, no. 4, pp. 913-923, 2013.

[19] N. Buls, G. Van Gompel, T. Van Cauteren et al., "Contrast agent and radiation dose reduction in abdominal CT by a combination of low tube voltage and advanced image reconstruction algorithms," European Radiology, vol. 25, no. 4, pp. 1023-1031, 2015.

[20] T. Van Cauteren, N. Buls, K. Tanaka, D. Verdries, G. Van Gompel, K. Nieboer et al., Reduced iodine dose in coronary CT angiography by combining low tube voltage acquisition 
with iterative reconstruction, European Society of Radiology; European Society of Cardiac Radiology, London, UK, 2013.

[21] T. Van Cauteren, N. Buls, K. Tanaka et al., "The impact of combining low-tube voltage scanning with iterative reconstruction on total iodine dose in coronary CT angiography," in Proceedings of the European Society of Radiology, European Congress of Radiology (ECR '14), Vienna, Austria, 2014.

[22] J. Leipsic, T. M. LaBounty, B. Heilbron et al., "Adaptive statistical iterative reconstruction: assessment of image noise and image quality in coronary CT angiography," American Journal of Roentgenology, vol. 195, no. 3, pp. 649-654, 2010.

[23] W. A. Clarkson, C. S. Restrepo, T. D. Bauch, and B. J. Rubal, "Using multidetector computed tomography in a swine model to assess the effects of sublingual nitroglycerin and intravenous adenosine on epicardial coronary arteries," Comparative Medicine, vol. 59, no. 6, pp. 573-579, 2009.

[24] Smith. C. P., Information Resources on Swine in Biomedical Research, AWIC Resource Series No. 11, United States Department of Agriculture, Beltsville, Md, USA, 1990-2000.

[25] A. W. Krazinski, F. G. Meinel, U. J. Schoepf et al., "Reduced radiation dose and improved image quality at cardiovascular CT angiography by automated attenuation-based tube voltage selection: intra-individual comparison," European Radiology, vol. 24, no. 11, pp. 2677-2684, 2014.

[26] T. Nakaura, K. Awai, Y. Yanaga et al., "Low-dose contrast protocol using the test bolus technique for 64-detector computed tomography coronary angiography," Japanese Journal of Radiology, vol. 29, no. 7, pp. 457-465, 2011.

[27] K. K. Kumamaru, M. L. Steigner, S. Soga et al., "Coronary enhancement for prospective ECG-gated single R-R axial 320MDCT angiography: comparison of 60- and $80-\mathrm{mL}$ iopamidol 370 injection," American Journal of Roentgenology, vol. 197, no. 4, pp. 844-850, 2011.

[28] W.-L. Zhang, M. Li, B. Zhang et al., "CT angiography of the head-and-neck vessels acquired with low tube voltage, low iodine, and iterative image reconstruction: clinical evaluation of radiation dose and image quality," PLoS ONE, vol. 8, no. 12, Article ID e81486, 2013.

[29] Y. Nakayama, K. Awai, Y. Funama et al., "Abdominal CT with low tube voltage: preliminary observations about radiation dose, contrast enhancement, image quality, and noise," Radiology, vol. 237, no. 3, pp. 945-951, 2005.

[30] M. J. Siegel, B. Schmidt, D. Bradley, C. Suess, and C. Hildebolt, "Radiation dose and image quality in pediatric CT: effect of technical factors and phantom size and shape," Radiology, vol. 233, no. 2, pp. 515-522, 2004.

[31] L. J. Zhang, L. Qi, J. Wang et al., "Feasibility of prospectively ECG-triggered high-pitch coronary CT angiography with $30 \mathrm{~mL}$ iodinated contrast agent at $70 \mathrm{kVp}$ : initial experience," European Radiology, vol. 24, no. 7, pp. 1537-1546, 2014.

[32] J. M. Racadio, S. R. Kashinkunti, R. A. Nachabe et al., "Clinically useful dilution factors for iodine and gadolinium contrast material: an animal model of pediatric digital subtraction angiography using state-of-the-art flat-panel detectors," Pediatric Radiology, vol. 43, no. 11, pp. 1491-1501, 2013.

[33] T. Choi, J. Malpeso, D. Li, S. Sourayanezhad, and M. J. Budoff, "Radiation dose reduction with increasing utilization of prospective gating in 64-multidetector cardiac computed tomography angiography," Journal of Cardiovascular Computed Tomography, vol. 5, no. 4, pp. 264-270, 2011.

[34] C. Mihl, J. E. Wildberger, T. Jurencak et al., "Intravascular enhancement with identical iodine delivery rate using different iodine contrast media in a circulation phantom," Investigative Radiology, vol. 48, no. 11, pp. 813-818, 2013.

[35] M. Kok, C. Mihl, A. Seehofnerová et al., "Automated tube voltage selection for radiation dose reduction in ct angiography using different contrast media concentrations and a constant iodine delivery rate," American Journal of Roentgenology, vol. 205, no. 6, pp. 1332-1338, 2015. 


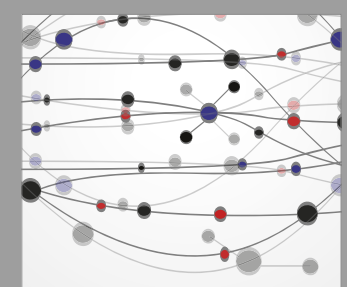

The Scientific World Journal
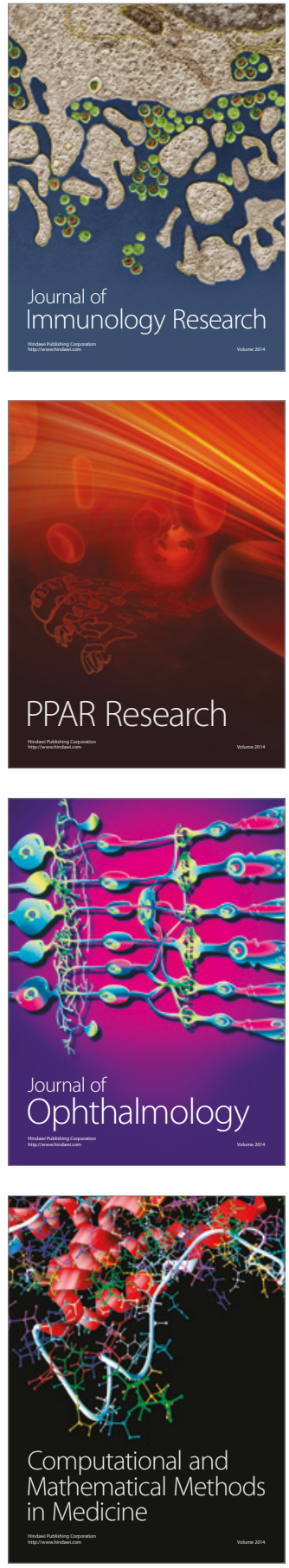

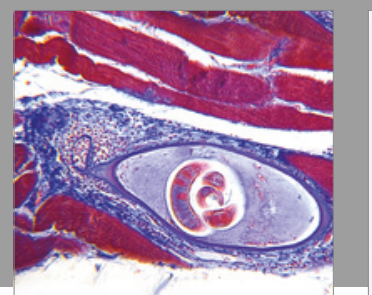

Gastroenterology Research and Practice
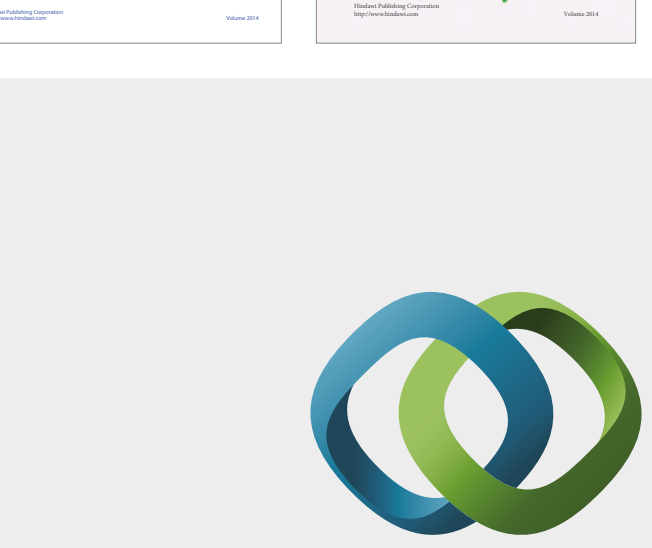

\section{Hindawi}

Submit your manuscripts at

https://www.hindawi.com
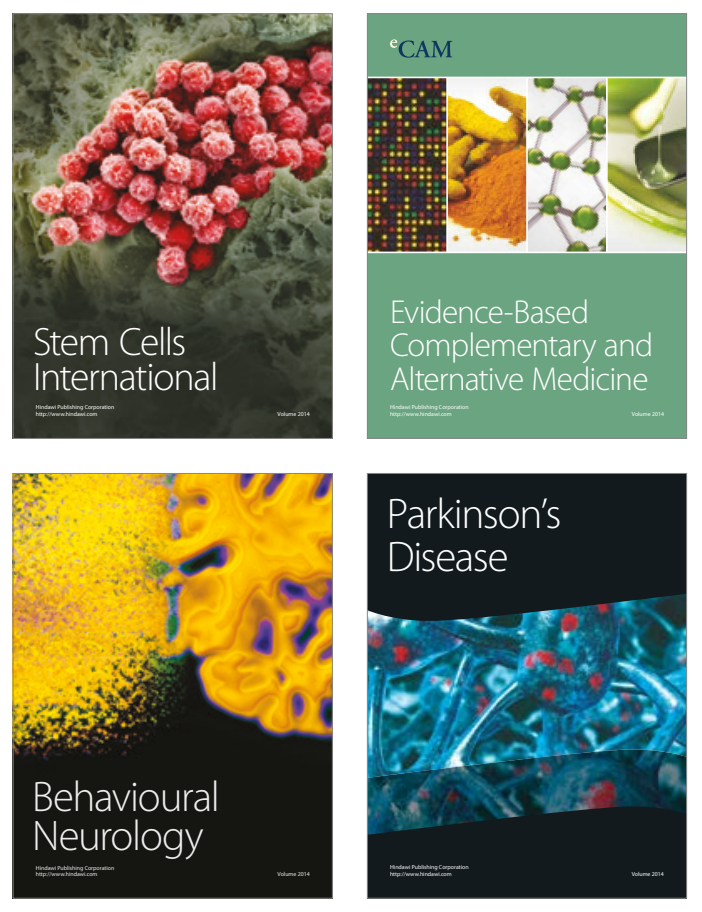
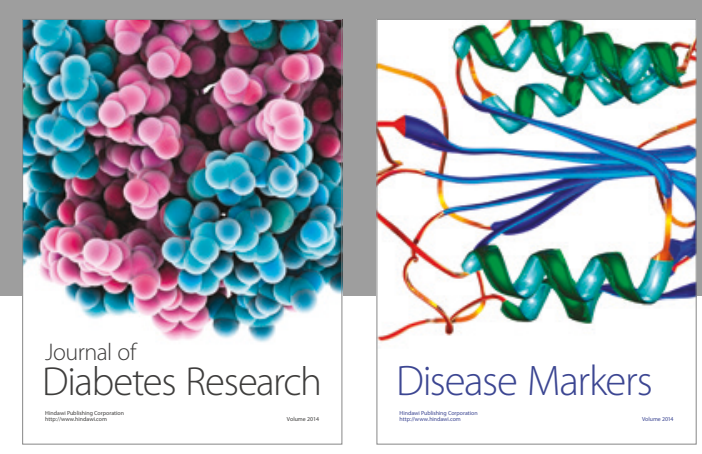

Disease Markers
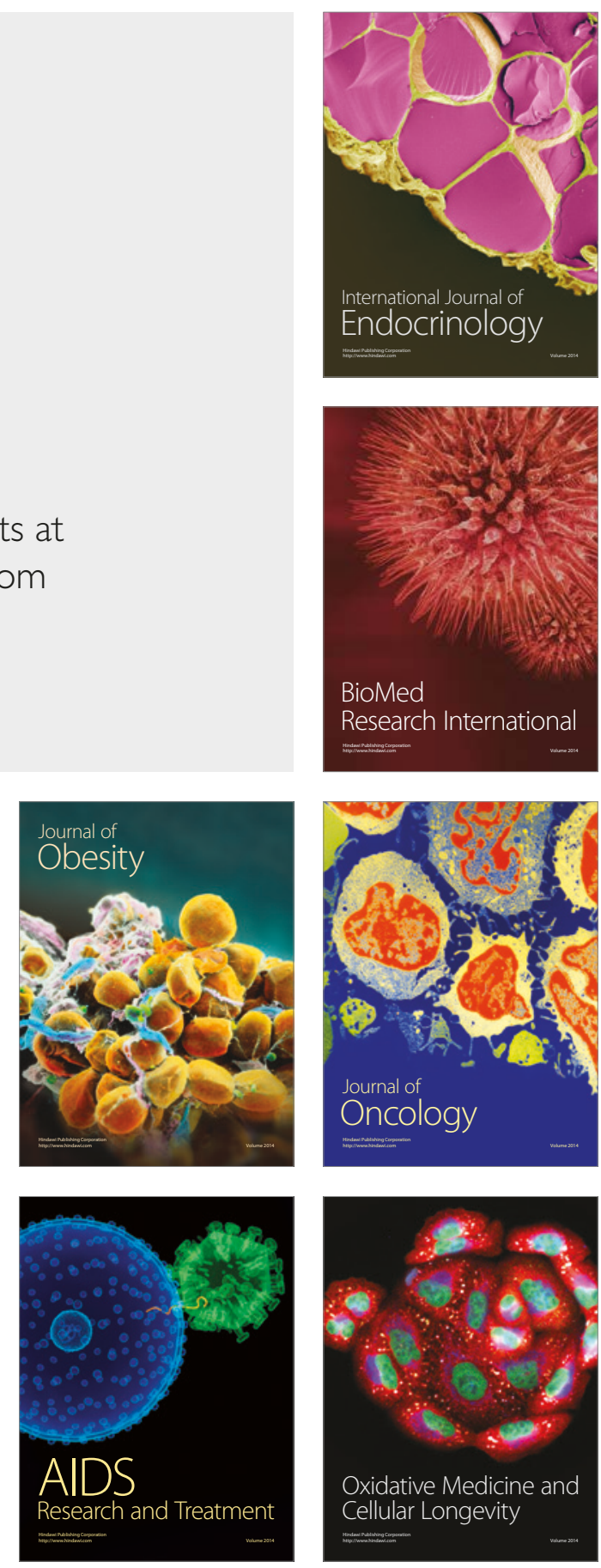